\title{
MANIFESTATION OF THE 160-min SOLAR OSCILLATIONS \\ IN VELOCITY AND BRIGHTNESS (OPTICAL AND RADIO OBSERVATIONS)*
}

\author{
(Invited Review) \\ V. A. KOTOV, A. B. SEVERNY, T. T. TSAP, \\ I. G. MOISEEV, V. A. EFANOV, and N. S. NESTEROV \\ Crimean Astrophysical Observatory, P.O. Nauchny, Crimea 334413, U.S.S.R.
}

\begin{abstract}
All evidence of the solar origin of 160 -min period oscillations is collected, and the present state of observations of this oscillation in optical and radio-ranges is considered. The main results are summarized: (a) the 160-min oscillation was observed in 1981 as well as before, (b) an attempt to find a nonradial component with $l=2$ has failed, (c) the intensity and circular polarization of radioemission show with statistical significance the presence of this 160 -min periodicity.
\end{abstract}

\section{On the Solar Origin of $\mathbf{1 6 0}-\mathrm{min}$ Oscillations}

The 160-min periodicity has the same phase at different sites of the Earth's globe: Crimea, Stanford, and South Pole (see Figure 1); the last observations lasting for about 5 days without interruptions caused by night-time intervals as is inevitable in usual ground-based observations show also that the 1-day sampling effect does not play any role. Moreover, the 160-min peak in the power spectrum (computed for 1974-1980) is the highest (see Figure 2). A possible influence of the differential transparency of the terrestrial atmosphere (difference in transparency between east and west limbs) can, in principle, bring some modulation, but this effect, on average, was reported to be at least 10 times smaller than the observed one (see Severny et al., 1980) and does not produce the systematic shift of the phase from year to year. Besides that, the changes of transparency do not show the 160 -min variations, as follows from the work by Clarke (1980). The apparent absence of the 160-min oscillations in velocity when one uses a telluric line, as well as the indication for the 27.2-day periodicity in the variations of an amplitude of this 160-min oscillation (Kotov et al., 1982), are also worth noticing. One should also note that the synchronous, in parallel with the line-of-sight velocity, variations in the intensity and circular polarization of radioemission from the Sun are observed (see below), which can not be ascribed to fluctuations in the ionosphere of the Earth. The 160-min variations in IR limb-darkening were observed by Koutchmy's and Kotov (1980). A full analysis of possible non-solar sources and errors is in Kotov et al. (1982).

\footnotetext{
* Proceedings of the 66th IAU Colloquium: Problems in Solar and Stellar Oscillations, held at the Crimean Astrophysical Observatory, U.S.S.R., 1-5 September, 1981.
}

Solar Physics 82 (1983) 9-19. 0038-0938/83/0821-0009\$01.65.

Copyright (C 1983 by D. Reidel Publishing Co., Dordrecht, Holland, and Boston, U.S.A. 


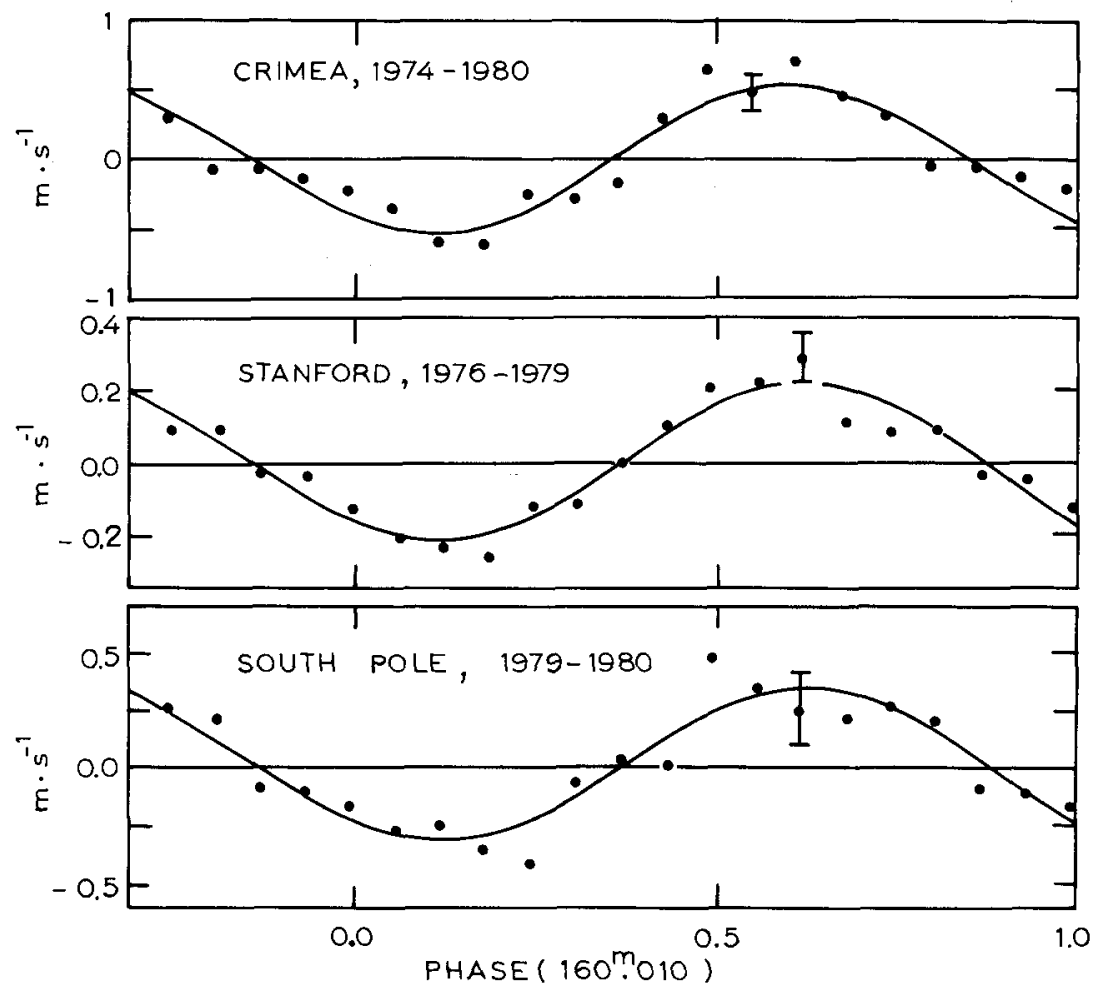

Fig. 1. Superposed epoch plot of the Crimean, Stanford, and South Pole observations with a folding period of $160.010 \mathrm{~min}$. Zero phase corresponds to UT $00^{\mathrm{h}} 00^{\mathrm{m}}$ on 1 January, 1974. The sinusoids represent the best fitted harmonic waves computed for three observatories separately. Vertical lines indicate rms error for each point.

\section{Present State of Optical Observations (1974-1980)}

A summary of the Crimean measurements of solar velocity is presented in Table I.

The results of superposed epoch analysis for the Crimea, Stanford, and South Pole are illustrated by Figure 1 showing good agreement in phases of mean line-of-sight velocity curves; some difference in amplitudes can be easily ascribed to the differences in methodes of measurements (different areas for averaging of velocity over the solar disk) and, in some part, - to difference in calibration. There is also a good agreement between the dependences of the moments of observed maximum (of outward velocity) upon the time (year) of observation (Figure 3); both Crimea and Stanford clearly show a year-to-year progressive drift of this moment provided that the period of the oscillations, in superposed epoch plots, is precisely $160.000 \mathrm{~min}$; this phase drift is found to be in quite a good agreement with South Pole result too (see label $\mathbf{N}$ on Figure 3). This drift of the velocity maximum, determined for each individual year with exactly $160.000 \mathrm{~min}$ period, points to a true period of $160.010 \mathrm{~min}$, corresponding to a yearly shift of the phase by about $32 \mathrm{~min}$, on average. 


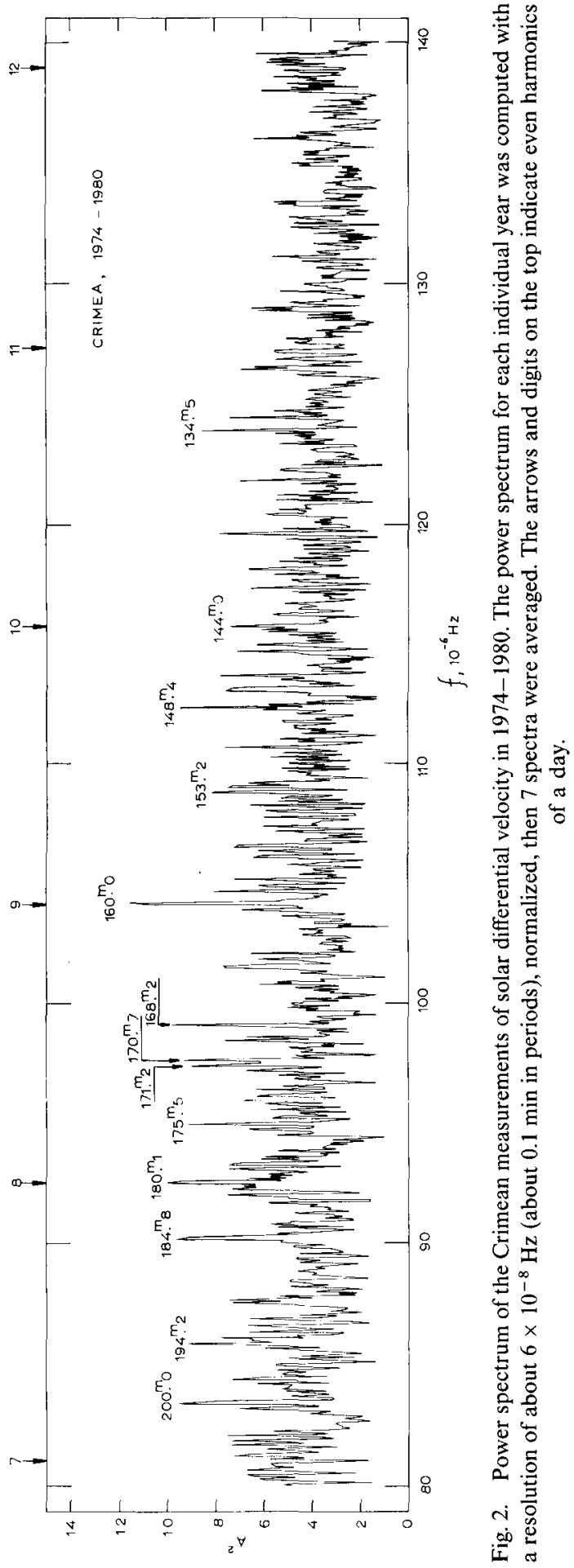




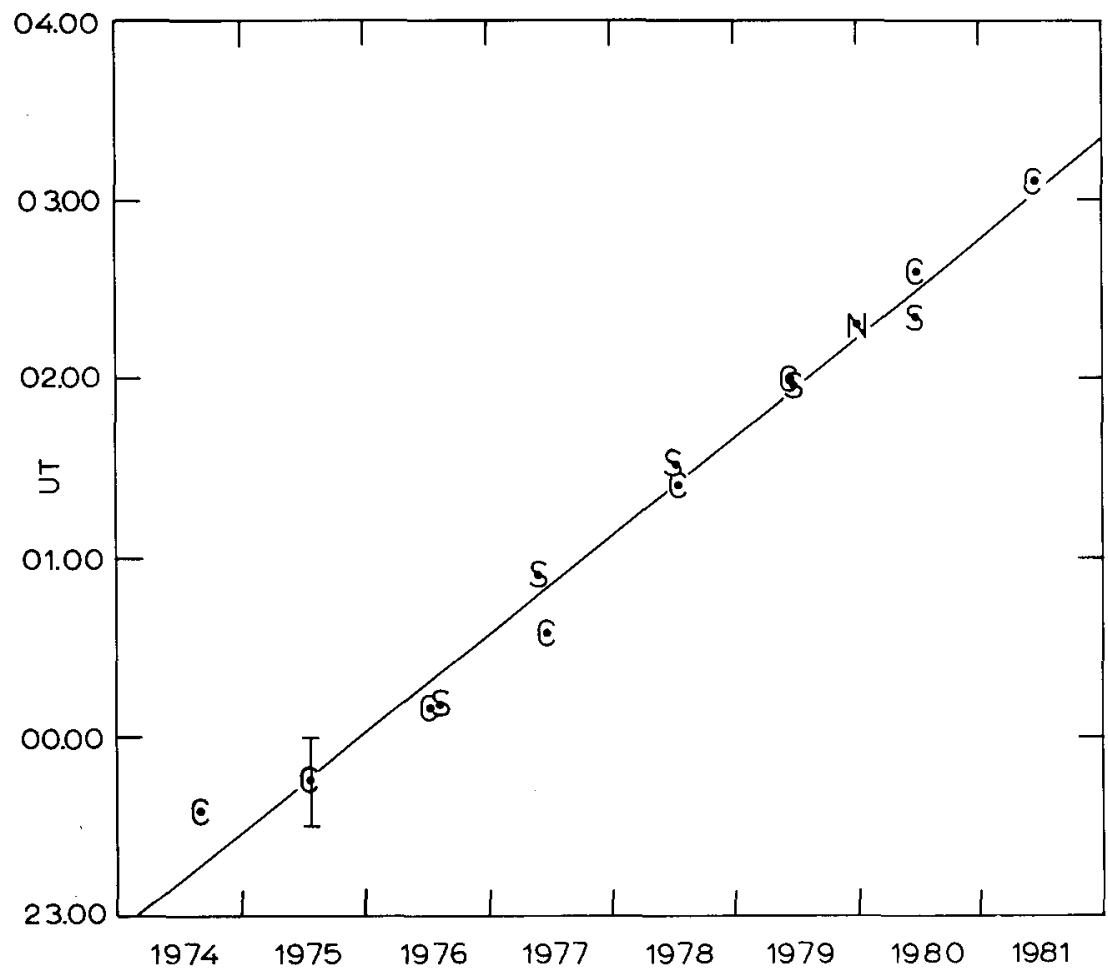

Fig. 3. Phase-shift diagram showing the progressive drift of the velocity maximum, by about 32 min per year, according to observations from different sites: C-Crimea, S - Stanford, N-geographic South Pole (see Scherrer et al., 1980; Grec et al., 1980). The phase shift was computed with respect to a $160 \mathrm{~min}$ period.

\section{TABLE I}

Summary of Crimean observations of solar velocity

\begin{tabular}{llllll}
\hline Year & Dates & Days & Hours & $\begin{array}{l}\text { Harmonic } \\
\text { amplitude } \\
\left(\mathrm{m} \mathrm{s}^{-1}\right)\end{array}$ & $\begin{array}{l}\text { UT } \\
\text { maximum } \\
\text { velocity }\end{array}$ \\
\hline 1974 & 4 August - 9 October & 14 & 76 & 1.42 & $23^{\mathrm{h}} 35^{\mathrm{m}}$ \\
1975 & 16 March - 14 October & 32 & 171 & 0.81 & $23^{\mathrm{h}} 45^{\mathrm{m}}$ \\
1976 & 26 April - 15 October & 32 & 163 & 0.36 & $00^{\mathrm{h}} 09^{\mathrm{m}}$ \\
1977 & 11 March - 24 October & 59 & 280 & 0.78 & $00^{\mathrm{h}} 35^{\mathrm{m}}$ \\
1978 & 22 April - 21 October & 78 & 471 & 0.48 & $01^{\mathrm{h}} 24^{\mathrm{m}}$ \\
1979 & 20 March - 30 December & 60 & 381 & 0.56 & $01^{\mathrm{h}} 59^{\mathrm{m}}$ \\
1980 & 14 January - 26 July & 50 & 304 & 0.45 & $02^{\mathrm{h}} 36^{\mathrm{m}}$ \\
1981 & 17 June - 31 July & 37 & 248 & 0.55 & $03^{\mathrm{h}} 07^{\mathrm{m}}$ \\
\hline Total & 4 August 1974 - 31 July 1981 & 362 & 2094 & - & - \\
\hline
\end{tabular}

${ }^{\text {a }}$ UT means the time of maximal velocity with respect to zero moment taken at UT $00^{\mathrm{h}} 00^{\mathrm{m}}$; superposed epoch analysis for each year is made with the period $160.000 \mathrm{~min}$. 


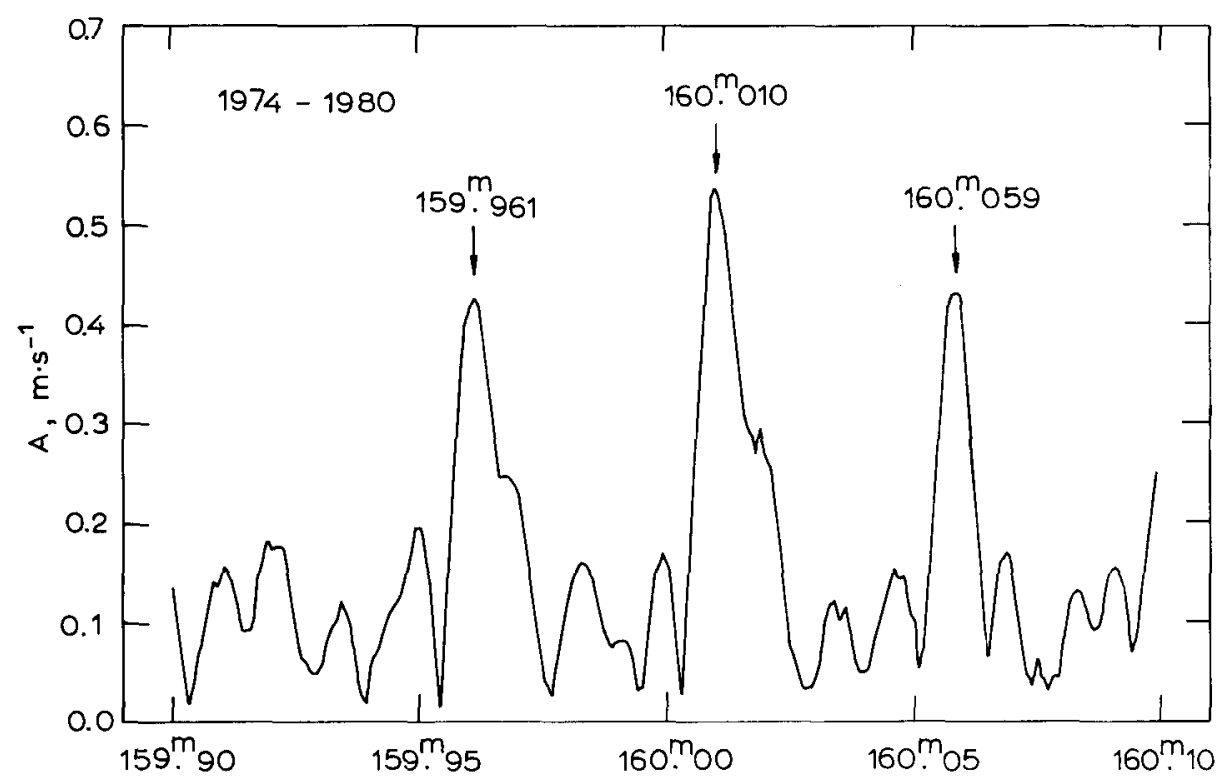

Fig. 4. High resolution power spectrum of the Crimean 1974-1980 measurements calculated near 160-min period (amplitude, in $\mathrm{m} \mathrm{s}^{-1}$, versus period, in $\mathrm{min}$ ).

There is no power in the velocity data at exactly $1 / 9$ th of a day (=160 min), as demonstrated by fine power spectrum on Figure 4; here the dominating peak is definitely located at the 160.010 min period, with two side bands produced by annual regularity of observational seasons. Comparison of the power spectra of long-period oscillations obtained in the Crimea (1974-1979) and at Stanford (1977-1980) shows good agreement of two observatories, especially with regard to the appearance of this 160 -min oscillation (see the paper of Scherrer and Wilcox, 1983). However, in addition to the common and dominant maximum at the 160 -min period, one can see other significant maxima. Particularly, in the power spectrum of the Crimean data 1974-1980 shown in Figure 2, the periods near 134, 148, 168, and 171 min (besides, of course, the artifact periods near $144 \mathrm{~min}$ and $180 \mathrm{~min}$ which are side bands of the $160.010 \mathrm{~min}$ period) are seen. When considering all these maxima one should keep in mind that in addition to some Earth-originated peaks, we may have also the transient, irregular effects of supergranulation and the newly discovered phenomenon of 'mesagranulation' (with a characteristic life-time of several hours) superposed on global oscillations of the Sun (November et al., 1981).

\section{Velocity Observations in 1981}

The persistent 160-min oscillation was observed again in 1981, as may be judged from Figure 3 where the phase (more precisely: initial phase) of maximal velocity according to reduction of the 1981 year observations agrees nicely with regression line computed 


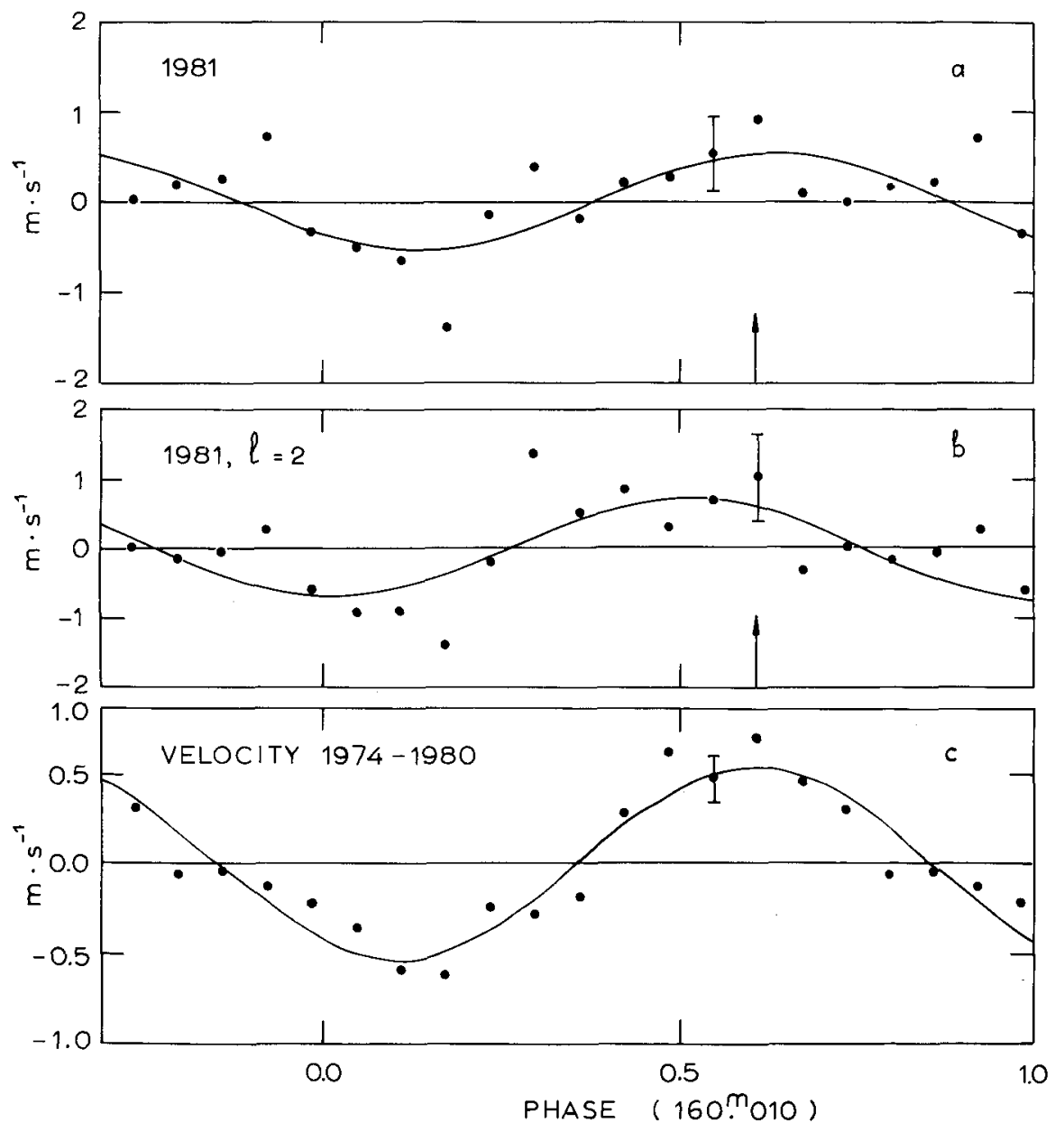

Fig. 5. (a) Superposed epoch plot of the total set of the velocity observations in 1981 including July measurements intended to look for $l=2$ oscillations (see Figure 6b). (b) Result of July measurements for detection of quadrupols $(l=2)$ motion. (c) Mean velocity curve according to previous, 1974-1980, Crimean observations. Vertical arrows show the position of velocity maxima predicted for 1981 on the basis of 1974-1980 observations.

from previous observations in 1974 to 1980 , and implying a 160.010 min period. The superposed epoch plot of these measurements made during 37 days in the summer of 1981 (from 17 June through $31 \mathrm{July}, 248 \mathrm{hr}$ in total) is shown in Figure 5 (top). The phase of the best-fitted harmonic curve corresponds to UT $01^{\mathrm{h}} 41^{\mathrm{m}}$, being in close agreement with the previous Crimean observations 1974 through $1980\left(\right.$ UT $01^{\mathrm{h}} 37^{\mathrm{m}}$ ), and Stanford measurements in $1976-1979\left(\right.$ UT $\left.01{ }^{\mathrm{h}} 37^{\mathrm{m}}\right)$, as well as with the observation at the South geographic pole (UT $01^{\mathrm{h}} 41^{\mathrm{m}}$; Grec et al., 1980); zero phase everywhere is taken at UT $00^{\mathrm{h}} 00^{\mathrm{m}}, 1 \mathrm{~J}$ anuary, 1974. 


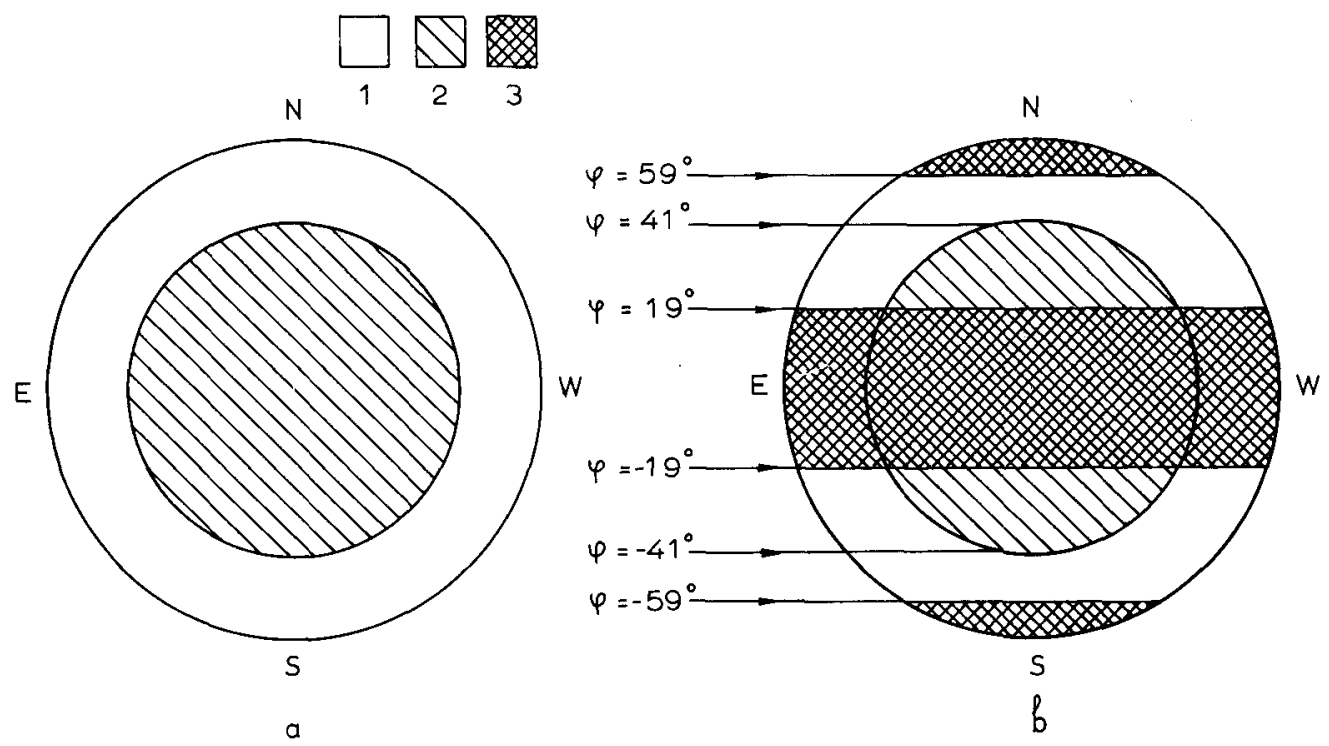

Fig. 6. A sketch showing the different areas of the solar disk used in the Crimea to measure differential solar velocity. (a) For the usual observations in 1974-1981 when the instrument detects the difference between the outer annulus and a weighted full disk. The weighting is such that the outer annulus (unshaded area) has full transmission and the inner circular area (shaded area of $0.66 D_{\odot}$ in diameter) has approximately half transmission. (b) For July 1981 observations to detect a quadrupole motion on the solar surface, when the equatorial and polar regions were screened by an opaque shield. The numbers 1,2 , and 3 on top shows three different kinds of areas: 1 - non-screened area; 2 - the area covered by circular polarizer; 3 - completely screened area. By $\varphi$ we denote different solar latitudes.

Our understanding of the 160-min oscillation, together with other modes of long periods $\left(\gtrsim 1^{\mathrm{h}}\right)$ can suffer from a lack of knowledge about the spatial distribution of amplitudes. This year we made an attempt to determine the type of 160-min oscillation with the use of the same observational technique but with quite different, than earlier, averaging of the velocities over the solar disk. Namely, to look for the quadrupole mode $(l=2)$ given by harmonic $P_{2}^{\circ}(\cos \theta)$ having two nodes on the solar surface (at heliolatitudes $\varphi \approx 55^{\circ}$ and $\approx-55^{\circ}$ where the radial component of the velocity $V_{r}=0$ ), we screened three portions of the solar disk: two polar regions for $|\varphi|>59^{\circ}$ and an extensive equatorial region limited by $|\varphi|<19^{\circ}$, see Figure 6 . In other respects the optical geometry for measurements of the differential $\left(V_{c}-V_{l}\right)$ velocity was retained the same as in all previous Crimean observations (see Kotov et al., 1982).

In these new series of observations (performed from 11 to 31 July, 1981; in all 19 days, $129 \mathrm{hr}$ ) we measured the difference between the line-of-sight velocities of two large areas located near heliolatitudes $|\varphi| \approx 25^{\circ}$ and the rest of non-screened areas $59^{\circ}>|\varphi|>19^{\circ}$ (see Figure 6). The result of these observations is plotted in the middle of Figure 5; we see that in these measurements purposed to make discrimination between the radial and quadrupole - types of oscillation, almost the same 160-min wave 

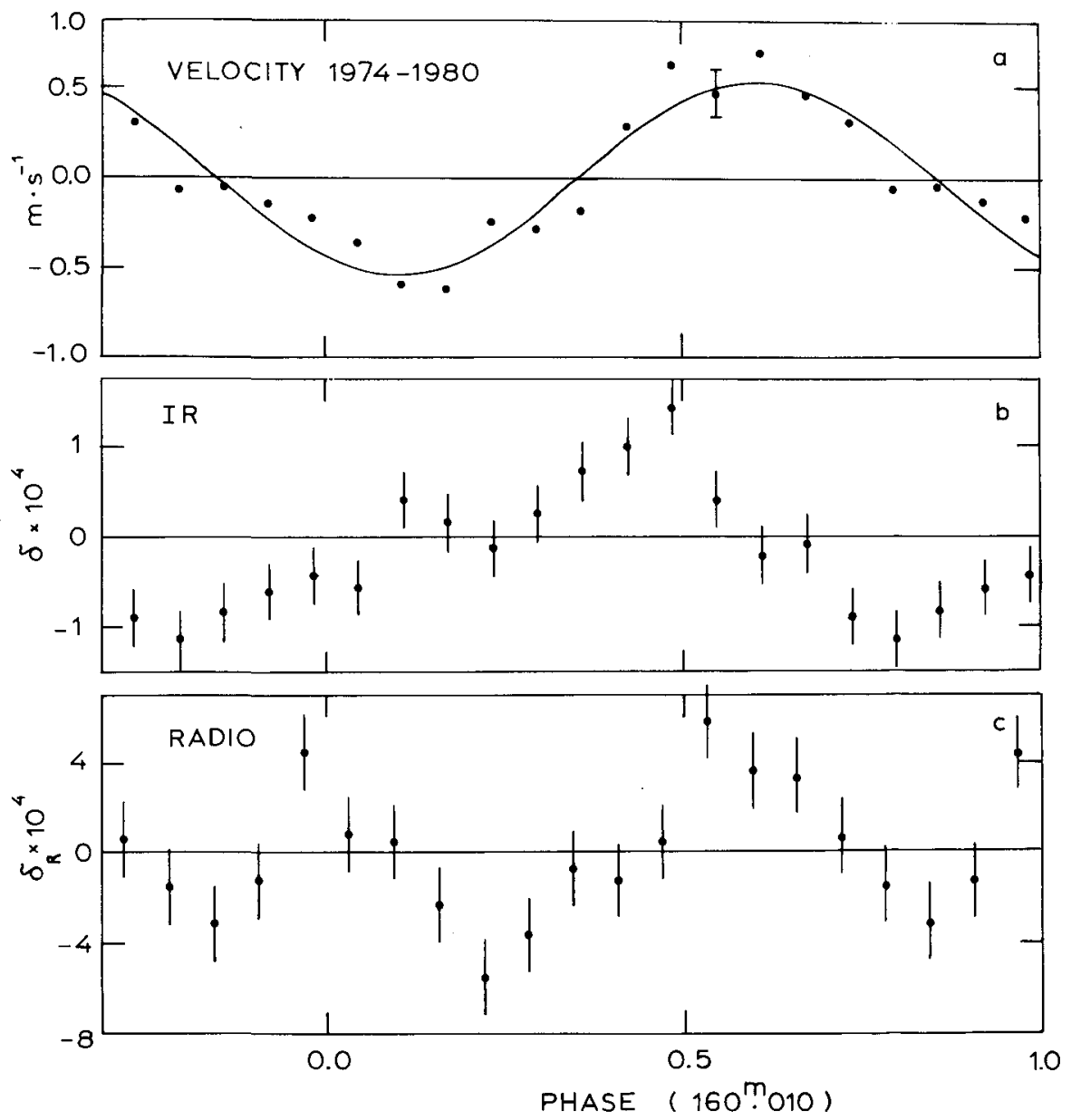

Fig. 7. Variations of velocity (a), IR brightness (b), and radioemission (c) with 160.010 min period. Phase zero is taken at UT $00^{\mathrm{h}} 00^{\mathrm{m}}, 1 \mathrm{January}, 1974$ (the same is for Figure 8).

has appeared as in the previous 1974-1980 observations (Figure 5, bottom). From this preliminary result one may conclude that 160 -min oscillation shows mostly radial motion.

\section{Radio Observations of 160 -min Oscillations}

To detect the changes in the center-to-limb difference of radiotemperature for the quiet Sun, and following the work by Eryushev et al. (1979), we measure alternately, with 5 -min integration time, the brightness temperature in two quiet regions of area of about 4 arc min square on the solar disk; one is selected near the disk center and the other is located about $10 \mathrm{arc}$ min to the north from the center. The first measurements of the ratio $\left(T_{l}-T_{c}\right) /\left(T_{l}+T_{c}\right)$ were made during 48 days in $1977-1980$ with the use of the Crimean 22-m dish antenna (see Ivanov et al., 1967) at four wavelengths: 1.9, 2.25, 2.5, 
and $3.5 \mathrm{~cm}$ (mostly at $2.25 \mathrm{~cm}$; in total about $400 \mathrm{hr}$ of measurements were collected). The first results were reported by Eryushev et al. (1979).

Diurnal drifts were eliminated by the usual procedure of 2 nd order polinomials approximation (similarly to the analysis of velocity records). The residuals then were subjected to superposed epoch analysis with the $160.010 \mathrm{~min}$ period according to the optical measurements.

Figure 7c shows the result of superposed epoch analysis for the difference $\delta_{R}=2\left(I_{l}-I_{c}\right) /\left(I_{l}+I_{c}\right)$ for the whole data set of 1977-1980. In the middle of Figure 7 we have plotted the mean curve of near infrared brightness changes according to measurements undertaken in 1977-1978 by Koutchmy et al. (1980), and at the top the mean velocity curve for 1974-1980; the phase was set to be 0 at UT $00^{\mathrm{h}} 00^{\mathrm{m}}$ on 1 January, 1974. Comparing all these curves one may conclude that there is an indication of the 160-min waves in solar intensity, IR and radio, with high statistical significance $(2 A / \sigma \gtrsim 5)$. The average power spectrum of the radio emission (not shown here) calculated for the total set of observations in 1977-1980 also exhibits a significant peak at $160^{\mathrm{m}}$.

There also exists a strong overtone with $80-90 \mathrm{~min}$ period in the differential (center-to-limb) radiobrightness. The delay in the phase of radio oscillations (see Figure 7) as compared with velocity variations might be caused by the time needed for the oscillations to propagate from the photosphere to the low corona; anyway, this question about time delay and correlation between the velocity and radio data might present a great interest for the theory called up to explain the 160 -min oscillations.

Measurements of polarization of the radioemission were made at $1.35 \mathrm{~cm}$ wavelength in June-July 1980 and June 1981, during 38 clear days in total. The degree of circular polarization was monitored successively in five different quiet regions on the solar disk: northern (N), central (C), southern (S), eastern (E), and western (W) locations, choosen far away from active centers. The Sun was observed for about $9 \mathrm{hr}$ per day on average (on each of 38 days in 1980 and 1981). Slow drifts again were subtracted from the data by best-fitting a parabola, and detrended residuals have formed a time series which have been processed using the superposed epoch technique and power spectrum analysis. Some data on the observations of these five locations and results - amplitudes and UT of observed maxima of polarization at the $160.010 \mathrm{~min}$ period - are given in Table II.

All five quiet regions on the Sun have shown the presence of statistically significant $(2 \mathrm{~A} / \sigma \approx 2-3)$ variations with the period $160.010 \mathrm{~min}$ and nearly with the same phase (see Table II, last column). The harmonic amplitude is about $10^{-5}$ (see Table II and Figure 8). The power spectrum averaged for these five sets of measurements of radio-polarization exhibited also a strong 160-min peak.

The polarization of the solar radioemission, as is well known, should be connected with the solar magnetic field. Hence, the 160-min variations of the degree of radio-polarization, shown in Figure 8 (bottom), might mean synchronous changes in the solar magnetic field. This offers a possibility to estimate the amplitude of the magnetic field variations. For the $\mathrm{cm}$-wavelength radioemission induced by thermal bremstrahlung there is a simple relation between degree of polarization and magnetic field strength (see 
TABLE II

Radio-polarization, $\lambda 1.35 \mathrm{~cm}, P=160.010 \mathrm{~min}$

(measurements in June-July 1980 and June $1981^{\text {a }}$ )

\begin{tabular}{llccccc}
\hline Location & \multicolumn{2}{l}{ Number of } & & $A \times 10^{5}$ & $2 A^{\prime} / \sigma$ & $t$ \\
\cline { 2 - 4 } & days & hours & $5^{\mathrm{m}}$-points & & & \\
\hline North & 26 & 235 & 1105 & 2.4 & 3.7 & $02^{\mathrm{h}} 10^{\mathrm{m}}$ \\
Center & 38 & 335 & 2122 & 1.2 & 4.2 & $01^{\mathrm{h}} 59^{\mathrm{m}}$ \\
South & 28 & 246 & 957 & 1.1 & 2.8 & $01^{\mathrm{h}} 54^{\mathrm{m}}$ \\
East & 13 & 104 & 688 & 1.0 & 2.2 & $01^{\mathrm{h}^{\mathrm{h}} 50^{\mathrm{m}}}$ \\
West & 3 & 28 & 182 & 5.4 & 3.8 & $02^{2^{\mathrm{h}} 00^{\mathrm{m}}}$ \\
\hline All & 38 & & 5054 & 1.5 & 6.9 & $02^{\mathrm{h}} 01^{\mathrm{m}}$
\end{tabular}

a $A$ denotes a harmonic amplitude, $2 A$ ' means 'peak-to-peak' amplitude (see Kotov et al., 1982); $t$ - time of maximal polarization in superposed epoch plots for $160.010 \mathrm{~min}$ period and zero moment taken at UT $00^{\mathrm{h}} 00^{\mathrm{m}}, 1$ January, 1974.
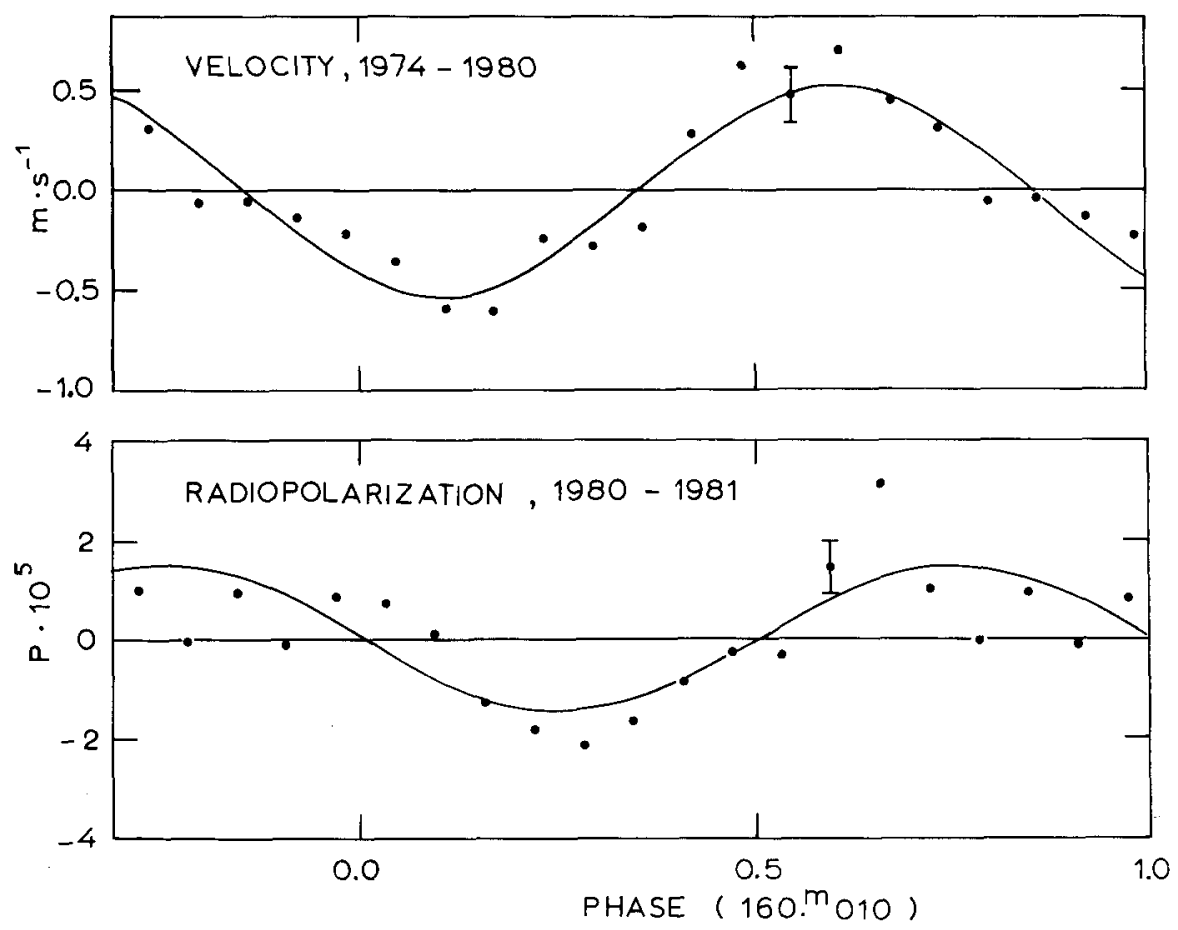

Fig. 8. Mean variations of the velocity and the degree of radio-polarization (at $\lambda 1.35 \mathrm{~cm}$ ) with the period of $160.010 \mathrm{~min}$. 
Bogod and Gelfreikh, 1980):

$$
p \approx n \frac{f_{H}}{f},
$$

where $n=\partial\left(\lg T_{b}\right) / \partial(\lg f)$ is the spectral index of radio-emission, $f_{H}=e H / 2 \pi \mathrm{cm}$ is the gyrofrequency, $f$ is the frequency of the observations and $H$ is the magnetic field strength. Substituting $\lambda=1.35 \mathrm{~cm}$ and $n \approx 1$, we get for the field strength:

$$
H \approx 0.79 \times 10^{4} p
$$

and for our mean value $p \approx 1.5 \times 10^{-5}$ the amplitude of magnetic field variations attains the value of about $0.12 \mathrm{G}$. This amplitude exceeds the directly estimated variations in the solar mean magnetic field inferred from optical observations (see Severny, 1969; Kotov et al., 1982) by a factor of $\approx 5$. However, this apparent discrepancy might be readily explained by the strongly inhomogeneous structure of the chromosphere and low corona, as well as by the fact that when one observes the Sun-as-a-star field one measures optically some average longitudinal component of the magnetic field for the solar disk as a whole, in contrast to the radio polarization measurements, where we deal with several small areas on the disk, and where the field strength may considerably exceed the mean field value.

It should be emphasized that the values of circular polarization and the amplitude of fluctuations of radioemission can not be attributed to ionospheric effects, where the optical depth for our wavelength $\left(1.35 \mathrm{~cm}\right.$ ) is about $5 \times 10^{-7}$ (for the measurements of radioflux) and $\sim 10^{-11}$ for the polarization.

\section{References}

Bogod, V. M. and Gelfreikh, G. B.: 1980, Solar Phys. 67, 29.

Clarke, D.: 1980, Monthly Notices Roy. Astron. Soc. 190, 641.

Eryushev, N. N., Kotov, V. A., Severny, A. B., and Tsvetkov, L. I.: 1979, Pis'ma v Astron. Zh. (USSR) 5, 546. Grec, G., Fossat, E., and Pomerantz, M.: 1980, Nature 288, 541.

Ivanov, V. N., Moiseev, I. G., and Monin, Y. G.: 1967, Izv. Krymsk. Astrofiz. Obs. 38, 141.

Kotov, V. A., Severny, A. B., and Tsap, T. T.: 1982, Izv. Krymsk. Astrofiz. Obs. 66, 3.

Koutchmy, S., Koutchmy, O., and Kotov, V. A.: 1980, Astron. Astrophys. 90, 372.

November, L. J., Toomre, J, Gebbie, K. B., and Simon, G. W.: 1981, Astrophys. J. 245, L123.

Scherrer, P. H. and Wilcox, J. M.: 1983, Solar Phys. 82, 37 (this volume).

Scherrer, P. H., Wilcox, J. M., Severny, A. B., Kotov, V. A., and Tsap, T. T.: 1980, Astrophys. J. 237 , L97.

Severny, A.: 1969, Nature 224, 53.

Severny, A. B., Kotov, V. A., and Tsap, T. T.: 1980, Astron. Astrophys. 88, 317. 